\title{
The Universal Structure of Local Algebras
}

\author{
D. Buchholz ${ }^{1}$, C. D'Antoni ${ }^{2, \star \star}$, and K. Fredenhagen ${ }^{1, \star}$ \\ ${ }^{1}$ II. Institut für Theoretische Physik, Universität Hamburg, D-2000 Hamburg 50, Federal \\ Republic of Germany \\ 2 Dipartimento di Matematica, Università di Roma, La Sapienza, Rome, Italy
}

\begin{abstract}
It is shown that a few physically significant conditions fix the global structure of the local algebras appearing in quantum field theory: it is isomorphic to that of $\Re \bar{\otimes} 3$, where $\mathfrak{R}$ is the unique hyperfinite factor of type $I I I_{1}$ and 3 the center of the respective algebra. The argument is based on results in $[1,2]$ relating to the type of the local algebras and an improvement of an argument in [3] concerning the "split property."
\end{abstract}

\section{Introduction}

Since the very beginnings of the algebraic approach to quantum field theory [4], there has been continuous interest in the structure of the local algebras appearing in this setting. This interest originates from the insight that the entire physical information of a quantum field theory is encoded in the map

$$
\mathcal{O} \rightarrow \mathfrak{A}(\mathcal{O})
$$

assigning to each bounded region $\mathcal{O}$ of Minkowski space a von Neumann algebra $\mathfrak{A}(\mathcal{O})$ which is generated by the observables (respectively fields) associated with the region in question. So there naturally arises the question of the concrete algebraic properties of the images $\mathfrak{A}(\mathcal{O})$ of this map.

It is by now well known that the local algebras $\mathfrak{A}(\mathcal{O})$ are, in generic cases, of type $I I I_{1}$ according to the classification of Connes (cf. [5]). This fact has been established in several models by explicit calculations, and also by more abstract arguments (cf. [6] for a review). But only recently this result has been derived from conditions which seem to be sufficiently general to cover most theories of physical interest [1]. Besides the standard postulates of quantum field theory the only input needed is the assumption that the theory has a scaling limit. This is expected to be the case in renormalizable field theories with an ultraviolet fixed point, hence in particular in all theories which are asymptotically free.

$\star$ Heisenberg fellow

$\star \star$ Research supported by Ministro della Pubblica Istruzione and CNR-GNAFA 
Although this result substantially restricts the possible structure of the local algebras it does not fix it completely since there exist non-isomorphic factors of type $I I I_{1}$. But, as was recently shown by Haagerup [2], there is only one such factor which is hyperfinite, i.e. generated by an increasing family of finite dimensional algebras.

The idea that the local algebras ought to be hyperfinite sounds physically reasonable and has been confirmed in models (cf. [6]). But it should be noticed that this is a quite subtle property. For example, a subalgebra of a hyperfinite algebra does not need to be hyperfinite. It is therefore gratifying that the hyperfiniteness of the local algebras can be derived, as we shall demonstrate, from gross properties having a simple physical interpretation.

Our starting point is the nuclearity condition proposed in [3] which restricts the number of local degrees of freedom of a theory in a sensible manner. We will show (Sect. 2) that theories satisfying this condition have the so-called "splitproperty" [7]. This means that for every pair of bounded regions $\mathcal{O}_{1}, \mathcal{O}_{2}$ with $\mathcal{O}_{1} \subset \subset \mathcal{O}_{2}$ (i.e. the closure of $\mathcal{O}_{1}$ has to be contained in the interior of $\mathcal{O}_{2}$ ) there exists some factor $\mathfrak{M}$ of type $I$ such that

$$
\mathfrak{U}\left(\mathcal{O}_{1}\right) \subset \mathfrak{M} \subset \mathfrak{A}\left(\mathcal{O}_{2}\right) .
$$

This result is an improvement on the somewhat weaker assertions in [3], saying that this inclusion holds if the region $\mathcal{O}_{2}$ is sufficiently large compared to $\mathcal{O}_{1}$ ("distal split property"). In the present context it is, however, essential that the boundaries of $\mathcal{O}_{1}$ and $\mathcal{O}_{2}$ may be arbitrarily close to each other. For this implies that the local algebras can be approximated from the inside (respectively from the outside) by type $I$ factors. As was pointed out in [8], it then follows that the local algebras are hyperfinite.

The only remaining ambiguity in the structure of the local algebras is the question of whether these algebras can have a center. It was argued in [4] that this possibility can be ruled out in the presence of equations of motion ("primitive causality"). In this case it would follow that the local algebras are isomorphic to the unique hyperfinite type $I I I_{1}$ factor $\Re[2]$. But a rigorous proof showing that the local algebras are factors does not yet exist.

In the presence of a center 3 the structure of the local algebras would, however, change only in a trivial manner. As will be discussed in Sect. 3, it then coincides with that of the tensor product of $\Re$ and 3 ,

$$
\mathfrak{A}(\mathcal{O}) \simeq \mathfrak{R} \bar{\otimes} 3 .
$$

So the results of this discussion may be summarized by saying that the local algebras in a quantum field theory exhibit the universal structure (1.3), whenever the number of local degrees of freedom of the theory complies with certain moderate limitations, and the underlying fields have a tame ultraviolet behavior. (The precise quantitative conditions are given in Sect. 3.) One may hope that this very explicit information on the local algebras will be the key to further progress in the structural analysis of the maps (1.1), in each of which the specific features of a particular theory are encoded. 


\section{Nuclearity and the Split Property}

We turn now to the central part of this investigation, namely the demonstration that the split property (1.2) holds in theories satisfying the nuclearity condition proposed in [3]. The consequences of this result will be discussed in the subsequent section.

Let us begin by specifying the assumptions entering into our argument.

1. (Net structure) We consider a net $\mathcal{O} \rightarrow \mathfrak{A}(\mathcal{O})$ of von Neumann algebras on a Hilbert space $\mathscr{H}$ which is associated with the open, bounded regions $\mathcal{O}$ of Minkowski space and satisfies the condition of isotony:

$$
\mathfrak{A}\left(\mathcal{O}_{1}\right) \subset \mathfrak{A}\left(\mathcal{O}_{2}\right) \text { if } \mathcal{O}_{1} \subset \mathcal{O}_{2} .
$$

Specific information about spacelike commutation properties ("locality") is not required in the present section.

2. (Translations) On $\mathscr{H}$ there is a continuous, unitary representation $t \rightarrow U(t)$ of $\mathbb{R}$ implementing the time translations on Minkowski space, i.e.

$$
U(t) \mathfrak{A}(\mathcal{O}) U(t)^{-1} \subset \mathfrak{A}(\mathcal{O}+t \cdot e) .
$$

Here $e$ denotes the vector fixing the time direction. The generator $H$ of the time translations $U(t)$ has non-negative spectrum including the eigenvalue 0 with multiplicity one, and the eigenvector $\Omega$ corresponding to this eigenvalue is cyclic and separating for the algebras $\mathfrak{Q}(\mathcal{O})$.

3. (Nuclearity) Let $\mathcal{O}$ be any fixed bounded region. Then each member of the family of maps $\Theta_{\beta}, \beta>0$ of $\mathfrak{H}(\mathcal{O})$ into $\mathscr{H}$ given by

$$
\Theta_{\beta}(A)=e^{-\beta H} A \Omega, \quad A \in \mathfrak{A}(\mathcal{O})
$$

is assumed to be nuclear. This means that for each $\Theta_{\beta}$ there exists a sequence of vectors $\Phi_{i} \in \mathscr{H}$ and of linear functionals $\varphi_{i} \in \mathfrak{A}(\mathcal{O})^{*}$ such that $\sum_{i}\left\|\varphi_{i}\right\|\left\|\Phi_{i}\right\|<\infty$ and

$$
\Theta_{\beta}(\cdot)=\sum_{i} \varphi_{i}(\cdot) \Phi_{i}
$$

Moreover, defining the trace-norm of $\Theta_{\beta}$ by

$$
\left\|\Theta_{\beta}\right\|_{1}=\inf \sum_{i}\left\|\varphi_{i}\right\|\left\|\Phi_{i}\right\|,
$$

where the infimum is to be taken with respect to all vectors $\Phi_{i}$ and functionals $\varphi_{i}$ complying with the above conditions, we assume the bound for $\beta \searrow 0$,

$$
\left\|\Theta_{\beta}\right\|_{1} \leqq e^{\left(\beta_{0} / \beta\right)^{n}},
$$

where $\beta_{0}, n$ are positive constants (depending only on $\mathcal{O}$ ).

This third assumption is equivalent to the nuclearity condition proposed in [3]. The present formulation, suggested by R. Longo, turns out to be more convenient in our discussion. For a brief explanation of the physical significance of the nuclearity condition see the subsequent section.

Our goal is the following

Theorem 2.1. Let $\mathcal{O} \rightarrow \mathfrak{A}(\mathcal{O})$ be a net of von Neumann algebras with the properties listed above. Then there exists for any pair of open, bounded regions $\mathcal{O}_{1}, \mathcal{O}_{2}$ with 
$\mathcal{O}_{1} \subset C \mathcal{O}_{2}$ some type I factor $\mathfrak{M}$ such that

$$
\mathfrak{U}\left(\mathcal{O}_{1}\right) \subset \mathfrak{M} \subset \mathfrak{U}\left(\mathcal{O}_{2}\right) .
$$

For the proof of this theorem we need two auxiliary lemmas. The first one is a simple consequence of the nuclearity condition.

Lemma 2.2. Let $f(\omega), \omega \geqq 0$ be any continuous function such that for some $\varepsilon>0$,

$$
\sup _{\omega \geqq 0}|f(\omega)| e^{(2+\varepsilon) \cdot\left(\beta_{0} \omega\right)^{n / n+1}}<\infty,
$$

where $\beta_{0}, n$ are the constants appearing in the formulation of the nuclearity condition. Then the map $\Theta$ of $\mathfrak{U}(\mathcal{O})$ into $\mathscr{H}$ given by

$$
\Theta(A)=f(H) \cdot A \Omega, \quad A \in \mathfrak{U}(\mathcal{O})
$$

is nuclear. Moreover, there exists a sequence of vectors $\Psi_{i} \in \mathscr{H}$ and of ultraweakly continuous (normal) linear functionals $\psi_{i} \in \mathfrak{U}(\mathcal{O})_{*}$ such that

$$
\sum_{i}\left\|\psi_{i}\right\|\left\|\Psi_{i}\right\|<\infty \quad \text { and } \quad \Theta(\cdot)=\sum_{i} \psi_{i}(\cdot) \Psi_{i} .
$$

Proof. Let $E_{j}, j \in \mathbb{N}$ be the spectral projections of $H$ corresponding to the spectral values $\omega, j-1 \leqq \omega<j$. Then the maps $\Xi_{j}$ given by

$$
\Xi_{j}(A)=E_{j} \cdot A \Omega, \quad A \in \mathfrak{U}(\mathcal{O})
$$

satisfy for any $\beta>0$ the identity

$$
\Xi_{j}(\cdot)=E_{j} e^{\beta H} \cdot \Theta_{\beta}(\cdot),
$$

where $\Theta_{\beta}$ are the nuclear maps defined in (2.3). Since the operators $E_{j} \cdot e^{\beta H}$ on $\mathscr{H}$ are bounded, it is clear that each $\Xi_{j}$ is nuclear. Moreover, on the basis of (2.6) we obtain (for large $j$ ) the bounds on the trace norms

$$
\begin{aligned}
\left\|\Xi_{j}\right\|_{1} & \leqq \inf _{\beta>0}\left\|E_{j} e^{\beta H}\right\| \cdot\left\|\Theta_{\beta}\right\|_{1} \\
& \leqq \inf _{\beta>0} e^{\beta j+\left(\beta_{0} / \beta\right)^{n}} \leqq e^{2\left(\beta_{0} j\right)^{n / n+1}} .
\end{aligned}
$$

From this it follows that the map

$$
\Xi(\cdot)=\sum_{j} e^{-(2+\varepsilon)\left(\beta_{0} j\right)^{n / n+1}} \Xi_{j}(\cdot)
$$

is nuclear too for any $\varepsilon>0$, since the sum is absolutely convergent with respect to the trace norm. Introducing now on $\mathscr{H}$ the (unbounded) operator,

$$
u(H)=\sum_{j} e^{(2+\varepsilon)\left(\beta_{0} j\right)^{n / n+1}} E_{j},
$$
and using the facts that $E_{i} E_{j}=\delta_{i j} E_{i}$, as well as $\sum_{j} E_{j}=1$, we see that the given map $\Theta$
can be represented by

$$
\Theta(\cdot)=f(H) u(H) \cdot \Xi(\cdot) .
$$

But from the postulated behavior of the function $f(\omega)$ for large $\omega$ it follows that the operator $f(H) u(H)$ is bounded. Hence $\Theta$ is nuclear. 
Now let $\Psi_{i} \in \mathscr{H}$ and $\varphi_{i} \in \mathfrak{U}(\mathcal{O})^{*}$ be sequences of vectors and functionals, respectively, such that $\sum_{i}\left\|\varphi_{i}\right\|\left\|\Psi_{i}\right\|<\infty$ and

$$
\Theta(\cdot)=\sum_{i} \varphi_{i}(\cdot) \Psi_{i}
$$

As is well known (cf. [9, Chap. III.2]) every functional $\varphi_{i} \in \mathfrak{A}(\mathcal{O})^{*}$ can uniquely be decomposed into a normal part $\psi_{i} \in \mathfrak{U}(\mathcal{O})_{*}$ and a singular part $\sigma_{i} \in \mathfrak{A}(\mathcal{O})_{*}^{\perp}$, i.e. $\varphi_{i}=\psi_{i}+\sigma_{i}$, and $\left\|\varphi_{i}\right\|=\left\|\psi_{i}\right\|+\left\|\sigma_{i}\right\|$. Consequently $\Theta$ can be represented as sum of the maps,

$$
\Theta^{(n)}(\cdot)=\sum_{i} \psi_{i}(\cdot) \Psi_{i} \quad \text { and } \quad \Theta^{(s)}(\cdot)=\sum_{i} \sigma_{i}(\cdot) \Psi_{i},
$$

each of which is nuclear. Since $\mathfrak{U}(\mathcal{O})_{*}$ and $\mathfrak{U}(\mathcal{O})_{*}^{\perp}$ are norm-closed subspaces of $\mathfrak{A}(\mathcal{O})^{*}$, it is also clear that $\Theta^{(n)}$ is normal and that $\Theta^{(s)}$ is singular. But $\Theta=\Theta^{(n)}+\Theta^{(s)}$ is a normal map from $\mathfrak{A}(\mathcal{O})$ into $\mathscr{H}$ (equipped with the ultra-weak topology) as is obvious from its definition. Hence $\Theta^{(s)}=\Theta-\Theta^{(n)}$, being normal and singular, must be the zero map.

In the second lemma we establish an identity for the vacuum expectation values of certain specific operators. In the proof we rely only on the assumed spectral properties of $H$.

Lemma 2.3. Let $\delta>0$. Then there exists some continuous function $f(\omega), \omega \in \mathbb{R}$ which decreases almost exponentially, i.e.

$$
\sup _{\omega}|f(\omega)| e^{|\omega|^{\kappa}}<\infty \quad \text { for any } 0<\kappa<1
$$

and which has the property that for any pair of bounded operators $A, B$ satisfying $\left[U(t) A U(t)^{-1}, B\right]=0$ for $|t|<\delta$, there holds the identity

$$
(\Omega, A B \Omega)=(\Omega, A f(H) B \Omega)+(\Omega, B f(H) A \Omega) .
$$

Proof. Let $\tau>0$ and let $\mathscr{P}_{\tau}=\mathbb{C} \backslash\{z: \operatorname{Im} z=0,|\operatorname{Re} z| \geqq \tau\}$ be the two-fold cut complex plane with cuts starting at $z= \pm \tau$. Making use of the invariance of $\Omega$ under the action of $U(t)$, the positivity of $H$, and the commutation properties of $A, B$, it follows from standard arguments that there exists on $\mathscr{P}_{\delta}$ some analytic function $h(z)$ such that for $t \in \mathbb{R}$,

$$
\lim _{\varepsilon \searrow 0} h(t+i \varepsilon)=(\Omega, A U(t) B \Omega)
$$

and

$$
\lim _{\varepsilon \searrow 0} h(t-i \varepsilon)=(\Omega, B U(-t) A \Omega)
$$

Now let $w \rightarrow z=2 \tau w /\left(w^{2}+1\right)$ be the conformal mapping of the unit disk $|w|<1$ onto the cut plane $\mathscr{P}_{\tau}$. If $0<\tau<\delta$, we have $\mathscr{P}_{\tau} \subset \mathscr{P}_{\delta}$, and consequently the function $w \rightarrow h\left(2 \tau w /\left(w^{2}+1\right)\right)$ is analytic on $|w|<1$. Its boundary values for $w \rightarrow e^{i \varphi}, 0 \leqq \varphi \leqq 2 \pi$ are

$$
(\Omega, A U(\tau / \cos \varphi) B \Omega) \quad \text { if } \quad 0 \leqq \varphi \leqq \pi, \quad \varphi \neq \pi / 2
$$


and

$$
(\Omega, B U(-\tau / \cos \varphi) A \Omega) \quad \text { if } \quad \pi \leqq \varphi \leqq 2 \pi, \quad \varphi \neq 3 \pi / 2 .
$$

The function may be discontinuous at $w= \pm i$, but it stays bounded at these points. In fact we have the uniform bound

$$
\left|h\left(2 \tau w /\left(w^{2}+1\right)\right)\right| \leqq\|A\| \cdot\|B\| \quad \text { for } \quad|w|<1,
$$

as is easily shown. Thus Cauchy's formula applies to this function even if we extend the path of integration to the boundary of the disk $|w|<1$, giving in particular

$$
h(0)=\frac{1}{2 \pi} \int_{0}^{2 \pi} d \varphi \lim _{r>1} h\left(2 \tau r e^{i \varphi} /\left(1+r^{2} e^{2 i \varphi}\right)\right)
$$

If we rewrite this result in terms of the vacuum expectation values we obtain

$$
(\Omega, A B \Omega)=\frac{1}{2 \pi} \int_{0}^{\pi} d \varphi\{(\Omega, A U(\tau / \cos \varphi) B \Omega)+(\Omega, B U(\tau / \cos \varphi) A \Omega)\}
$$

and this relation holds for all $0<\tau<\delta$. Now we pick some testfunction $\tilde{g}(\tau)$ with support in $0<\tau<\delta$, whose Fourier transform $g(\omega)$ is almost exponentially decreasing (in the sense made precise in the statement of the lemma) and does not vanish at $\omega=0$. For a proof that such functions exist cf. [10]. If we multiply the above relation for the vacuum expectation values with $\tilde{g}(\tau)$ and integrate with respect to $\tau$ we arrive at the identity

$$
(\Omega, A B \Omega)=(\Omega, A f(H) B \Omega)+(\Omega, B f(H) A \Omega),
$$

where $f(\omega), \omega \in \mathbb{R}$ is given by

$$
f(\omega)=\frac{1}{2 \pi g(0)} \int_{0}^{\pi} d \varphi g(\omega / \cos \varphi) .
$$

This function is continuous and decreases almost exponentially, as one easily verifies. So the proof of the lemma is complete.

With this information we can turn now to the proof of the split property: let $\mathcal{O}_{a}, \mathcal{O}_{b}$ be two arbitrary open bounded regions such that $\mathcal{O}_{a} \subset C \mathcal{O}_{b}$, and let $\mathfrak{A}\left(\mathcal{O}_{a}\right) \odot \mathfrak{A}\left(\mathcal{O}_{b}\right)^{\prime}$ be the algebraic tensor product of $\mathfrak{A}\left(\mathcal{O}_{a}\right)$ and $\mathfrak{A}\left(\mathcal{O}_{b}\right)^{\prime}$ [the commutant of $\mathfrak{U}\left(\mathcal{O}_{b}\right)$ in $\left.\mathscr{B}(\mathscr{H})\right]$. We consider two representations of this tensor product: the first one, denoted by $\pi$, acts on $\mathscr{H}$ and is obtained by linear extension from

$$
\pi(A \odot B)=A \cdot B \quad \text { for } \quad A \in \mathfrak{U}\left(\mathcal{O}_{a}\right), \quad B \in \mathfrak{U}\left(\mathcal{O}_{b}\right)^{\prime} .
$$

This definition is obviously consistent, and it defines a *-representation of the tensor product since the operators in $\mathfrak{U}\left(\mathcal{O}_{a}\right)$ and $\mathfrak{U}\left(\mathcal{O}_{b}\right)^{\prime}$ commute. The second representation, denoted by $\pi_{p}$, acts on $\mathscr{H} \otimes \mathscr{H}$ and is fixed by

$$
\pi_{p}(A \odot B)=A \otimes B \quad \text { for } \quad A \in \mathfrak{U}\left(\mathcal{O}_{a}\right), \quad B \in \mathfrak{U}\left(\mathcal{O}_{b}\right)^{\prime} .
$$

For the proof of the split property we must show that these representations are equivalent.

To this end we consider the state $\omega$ on $\mathfrak{A}\left(\mathcal{O}_{a}\right) \odot \mathfrak{A}\left(\mathcal{O}_{b}\right)^{\prime}$ given by

$$
\omega(A \odot B)=(\Omega, \pi(A \odot B) \Omega)=(\Omega, A B \Omega) .
$$


We will demonstrate first that $\omega$ is normal relative to the representation $\pi_{p}{ }^{1}$ : since $\mathcal{O}_{a} \subset \subset \mathcal{O}_{b}$, there is some $\delta>0$ such that $U(t) \mathfrak{H}\left(\mathcal{O}_{a}\right) U(t)^{-1} \subset \mathfrak{A}\left(\mathcal{O}_{b}\right)$ for $|t|<\delta$, cf. relations (2.1) and (2.2). Hence there exists according to Lemma 2.3 some continuous, almost exponentially decreasing function $f$ such that

$$
\omega(A \odot B)=(\Omega, A f(H) B \Omega)+(\Omega, B f(H) A \Omega)
$$

for all $A \in \mathfrak{Q}\left(\mathcal{O}_{a}\right)$ and $B \in \mathfrak{U}\left(\mathcal{O}_{b}\right)^{\prime}$. With the help of the nuclearity condition, it is then easy to show that both functionals appearing on the right-hand side of this identity are normal relative to $\pi_{p}$. Let us consider first the linear functional $\xi$ on $\mathfrak{A}\left(\mathcal{O}_{a}\right) \odot \mathfrak{A}\left(\mathcal{O}_{b}\right)^{\prime}$ given by

$$
\xi(A \odot B)=(\Omega, B f(H) A \Omega) .
$$

Taking into account that $\mathcal{O}_{a}$ is bounded as well as the specific properties of $f$ it follows from Lemma 2.2 that the map $\Theta(A)=f(H) A \Omega, A \in \mathfrak{U}\left(\mathcal{O}_{a}\right)$ is nuclear. Thus we can represent $\xi$ as an infinite sum of functionals $\xi_{i}$ of the form

$$
\xi_{i}(A \odot B)=\psi_{i}(A) \cdot\left(\Omega, B \Psi_{i}\right),
$$

where $\psi_{i} \in \mathfrak{A}\left(\mathcal{O}_{a}\right)_{*}, \Psi_{i} \in \mathscr{H}$ and $\sum_{i}\left\|\psi_{i}\right\|\left\|\Psi_{i}\right\|<\infty$. So each $\xi_{i}$ is manifestly normal relative to $\pi_{p}$, and since $\left\|\xi_{i}\right\| \leqq\left\|\psi_{i}\right\| \cdot\left\|\Psi_{i}\right\|$, we also have $\sum_{i}\left\|\xi_{i}\right\|<\infty$. Hence $\xi$, being an absolutely convergent sum of normal functionals, is normal. The same considerations apply to the functional

$$
\left.\eta(A \odot B)=(\Omega, A f(H) B \Omega)=\overline{\left(\Omega, B^{*} \bar{f}(H) A^{*} \Omega\right.}\right),
$$

proving that $\omega=\xi+\eta$ is normal relative to $\pi_{p}$.

Now since $\Omega$ is cyclic and separating for the algebras $\mathfrak{A}(\mathcal{O})$, the vector $\Omega \otimes \Omega \in \mathscr{H} \otimes \mathscr{H}$ is cyclic for the commutant of $\pi_{p}\left(\mathfrak{H}\left(\mathcal{O}_{a}\right) \odot \mathfrak{H}\left(\mathcal{O}_{b}\right)^{\prime}\right)$ in $\mathscr{B}(\mathscr{H}) \otimes \mathscr{B}(\mathscr{H})$. Hence every state which is normal relative to $\pi_{p}$ can be represented by a vectorstate in this representation [11, Theorem 2.7.9]. In particular, there are vectors $\Omega_{p} \in \mathscr{H} \otimes \mathscr{H}$ such that

$$
\omega(C)=\left(\Omega_{p}, \pi_{p}(C) \Omega_{p}\right) \quad \text { for } \quad C \in \mathfrak{A}\left(\mathcal{O}_{a}\right) \odot \mathfrak{U}\left(\mathcal{O}_{b}\right)^{\prime} .
$$

From this it readily follows that the representations $\pi$ and $\pi_{p}$ are not disjoint. For the proof that they are even equivalent, one could then apply the general arguments expounded in [3]. But the more specific information provided by Eq. (2.14) also allows a simple direct proof of this fact.

The essential step is the demonstration that the normal extension of $\omega$ to the W*-tensor product $\mathfrak{A}\left(\mathcal{O}_{a}\right) \bar{\otimes} \mathfrak{A}\left(\mathcal{O}_{b}\right)^{\prime}$ is faithful. For this implies that the vector $\Omega_{p}$ in relation (2.14) can be chosen to be cyclic for $\pi_{p}[11$, Theorem 2.7.9]. Since $\Omega$ is cyclic for $\pi$ and since

$$
(\Omega, \pi(C) \Omega)=\left(\Omega_{p}, \pi_{p}(C) \Omega_{p}\right) \quad \text { for } \quad C \in \mathfrak{A}\left(\mathcal{O}_{a}\right) \odot \mathfrak{A}\left(\mathcal{O}_{b}\right)^{\prime},
$$

cf. relations (2.9) and (2.14), it is then clear that $\pi$ and $\pi_{p}$ are unitarily equivalent.

For the proof that $\omega$ is faithful we first recall that the choice of the regions $\mathcal{O}_{a}, \mathcal{O}_{b}$ in our discussion was completely arbitrary, apart from the condition that $\mathcal{O}_{a} \subset \subset \mathcal{O}_{b}$.

${ }^{1}$ A linear functional on $\mathfrak{A}\left(\mathcal{O}_{1}\right) \odot \mathfrak{A}\left(\mathcal{O}_{2}\right)^{\prime}$ is said to be normal relative to $\pi_{p}$ if it is continuous with respect to the ultra weak topology determined by this representation 
We may thus choose regions $\widetilde{\mathcal{O}}_{a}, \widetilde{\mathcal{O}}_{b}$ such that $\mathcal{O}_{a} \subset \subset \widetilde{\mathcal{O}}_{a} \subset \subset \widetilde{\mathcal{O}}_{b} \subset \subset \mathcal{O}_{b}$ and consider the representations $\tilde{\pi}, \tilde{\pi}_{p}$ of $\mathfrak{U}\left(\tilde{\mathcal{O}}_{a}\right) \odot \mathfrak{U}\left(\tilde{\mathcal{O}}_{b}\right)^{\prime}$, which are defined as in relations $(2.7)$ and (2.8), respectively. The corresponding state $\tilde{\omega}$, defined as in (2.9), is an extension of $\omega$ to the larger algebra under consideration. It is normal relative to $\tilde{\pi}_{p}$ and may thus be represented by some vector $\widetilde{\Omega}_{p} \in \mathscr{H} \otimes \mathscr{H}$. We note that, since $\Omega \otimes \Omega$ is separating for $\mathfrak{U}\left(\widetilde{\mathcal{O}}_{a}\right) \widetilde{\otimes} \mathfrak{U}\left(\widetilde{\mathcal{O}}_{b}\right)^{\prime}$, there is also such a vector $\widetilde{\Omega}_{p}$ which is not orthogonal to $\Omega \otimes \Omega$.

Now because of the choice of the regions $\widetilde{\mathcal{O}}_{a}, \widetilde{\mathcal{O}}_{b}$, relation $(2.2)$, and the fact that $\Omega$ is invariant under the action of $U(t)$ there exists some $\delta>0$ such that for all $C \in \mathfrak{A}\left(\mathcal{O}_{a}\right) \odot \mathfrak{A}\left(\mathcal{O}_{b}\right)^{\prime}$ and $|t|<\delta$,

$$
\omega(C)=\left(\widetilde{\Omega}_{p}, U_{p}(t) \tilde{\pi}_{p}(C) U_{p}(t)^{-1} \widetilde{\Omega}_{p}\right),
$$

where $U_{p}(t)=U(t) \otimes U(t)$. Hence if $X_{\imath} \in \mathfrak{U}\left(\mathcal{O}_{a}\right) \odot \mathfrak{A}\left(\mathcal{O}_{b}\right)^{\prime}$ is any net which converges strongly to some $X \in \mathfrak{A}\left(\mathcal{O}_{a}\right) \bar{\otimes} \mathfrak{A}\left(\mathcal{O}_{b}\right)^{\prime}$ and if $\lim \omega\left(X_{\imath}^{*} X_{\imath}\right)=0$, it follows from (2.16) that for $|t|<\delta$,

$$
X \cdot U_{p}(t) \widetilde{\Omega}_{p}=0 .
$$

Since the generator of $t \rightarrow U_{p}(t)$ is positive, this equation extends by analyticity to any $t \in \mathbb{R}$. Taking also into account that the multiples of $\Omega \otimes \Omega$ are the only vectors which are invariant under the action of $U_{p}(t)$, we thus find, by taking in (2.17) a suitable mean over $t$, that $X \cdot \Omega \otimes \Omega=0$. Hence $X=0$, so the normal extension of $\omega$ to $\mathfrak{U}\left(\mathcal{O}_{a}\right) \bar{\otimes} \mathfrak{A}\left(\mathcal{O}_{b}\right)^{\prime}$ is faithful, as claimed.

Having shown that the representations $\pi$ and $\pi_{p}$ are equivalent, the proof of the split property is now easily accomplished: let $V$ be any isometry mapping $\mathscr{H}$ onto $\mathscr{H} \otimes \mathscr{H}$ which establishes the equivalence of $\pi$ and $\pi_{p}$. We then have

$$
A \cdot B=V^{-1} A \otimes B V \quad \text { for } \quad A \in \mathfrak{A}\left(\mathcal{O}_{a}\right), \quad B \in \mathfrak{U}\left(\mathcal{O}_{b}\right)^{\prime} .
$$

Since $\mathfrak{N}=V^{-1} \mathscr{B}(\mathscr{H}) \otimes 1 V$ is clearly a type $I$ factor and since there holds the trivial inclusion

$$
V^{-1} \mathfrak{A}\left(\mathcal{O}_{a}\right) \otimes 1 V \subset V^{-1} \mathscr{B}(\mathscr{H}) \otimes 1 V \subset\left(V^{-1} 1 \otimes \mathfrak{A}\left(\mathcal{O}_{b}\right)^{\prime} V\right)^{\prime}
$$

we arrive at

$$
\mathfrak{U}\left(\mathcal{O}_{a}\right) \subset \mathfrak{N} \subset \mathfrak{U}\left(\mathcal{O}_{b}\right) .
$$

This completes our proof of the split property.

\section{Quantum Fields and the Structure of Local Algebras}

We will establish now the universal structure (1.3) of the local algebras in quantum field theory by combining information about the type of these algebras with the results of the previous section. Strictly speaking, the present section is nothing but a summary of known facts. But we found it worth-while to bring together here the scattered information which is relevant in the present context.

Our starting point is the standard formulation of local quantum field theory in terms of Wightman fields. Since this setting is well-known we can be very brief in stating the relevant assumptions. 
1. (Wightman axioms [12]) We consider a *-algebra $\mathfrak{P}$ of (unbounded) operators generated by a collection of Wightman fields $F_{\alpha}$ and acting on some domain in a Hilbert space $\mathscr{H}$. So the fields $F_{\alpha}$ are temperate distributions on Minkowski space with values in $\mathfrak{P}$ on which the Poincare group (respectively its covering group) $\mathscr{P}_{+}^{\uparrow}$ acts by automorphisms. These automorphisms are implemented on $\mathscr{H}$ by a continuous, unitary representation $L \rightarrow U(L)$ of $\mathscr{P}_{+}^{\dagger}$. In connection with the question of the type of the local algebras, it will be essential that the fields are finitely covariant, i.e. that each $F_{\alpha}$ transforms under the subgroup of Lorentz-transformations according to some finite-dimensional representation. The fields $F_{\alpha}$ are local and satisfy normal spacelike commutation, respectively anticommutation relations. At last, there is an (up to a phase unique) unit vector $\Omega$ which is invariant under the action of the unitaries $U(L)$ and which is the ground state of the Hamiltonian $H$ (the generator of the time-translations). We assume that $\Omega$ is cyclic for the algebra $\mathfrak{P}$ and that the resulting domain $\mathscr{D}=\mathfrak{B} \Omega$ is a core for the field operators $F_{\alpha}(f)$ for any testfunction $f$.

This setting is familiar from many field-theoretic models. We emphasize that it also applies to gauge quantum field theory if one restricts the algebra $\mathfrak{P}$ to local, gauge-invariant fields and observables, respectively.

Given this structure, we can define a Poincaré-covariant net $\mathcal{O} \rightarrow \underline{\mathfrak{A}}(\mathcal{O})$ of von Neumann algebras on $\mathscr{H}$ as follows: let $\mathcal{O}$ be any open, bounded region in Minkowski space and let $\mathscr{S}(\mathcal{O})$ be the subspace of all testfunctions with support in $\mathcal{O}$. Picking any field $F_{\alpha}$ and any $f \in \mathscr{S}(\mathcal{O})$, we obtain a field operator $F_{\alpha}(f) \in \mathfrak{P}$ which is associated with the region $\mathcal{O}$. This operator, being an element of a ${ }^{*}$-algebra, has a densely defined adjoint, and hence is closable. It thus determines in a canonical way the algebra $\mathfrak{R}_{\alpha, f}=\left\{F_{\alpha}(f)^{*}, F_{\alpha}(f)^{* *}\right\}^{\prime \prime}$, which is the smallest von Neumann algebra to which the closure of $F_{\alpha}(f)$ is affiliated. We then define $\mathfrak{Y}(\mathcal{O})$ as the von Neumann algebra generated by all $\mathfrak{R}_{\alpha, f}$ with $f \in \mathscr{S}(\mathcal{O})$,

$$
\underline{\mathfrak{I}}(\mathcal{O})=\bigvee_{\alpha, f \in \mathscr{S}(\mathcal{O})} \Re_{\alpha, f} \text {. }
$$

It follows immediately from this definition that the family of algebras $\mathfrak{a}(\mathcal{O})$ so defined satisfies the condition of isotony, cf. relation (2.1). Moreover, since the support of a function is a closed set (by definition), the algebras $\mathfrak{Y}(\mathcal{O})$ are "continuous from the inside," i.e. for each increasing family of open regions $\mathcal{O}_{i}$ with $\bigcup_{i} \mathcal{O}_{i}=\mathcal{O}$, we have

$$
\underline{\mathfrak{X}}(\mathcal{O})=\bigvee_{i} \mathfrak{\mathscr { A }}\left(\mathcal{O}_{i}\right)
$$

From the Poincare covariance of the fields $F_{\alpha}$, it is also clear that the Poincare transformations act on the algebras $\mathfrak{Q}(\mathcal{O})$ in the geometrically obvious manner

$$
U(L) \mathfrak{X}(\mathcal{O}) U(L)^{-1} \subset \mathfrak{Y}(L \mathcal{O}) .
$$

The only feature which does not immediately carry over from the Wightman fields to the corresponding net of von Neumann algebras is the property of locality. Disregarding the fact that the field operators are unbounded one would deduce from the spacelike commutation relations of the fields the following specific structure of the algebras $\mathfrak{Y}(\mathcal{O})$ : each operator $C \in \mathfrak{Y}(\mathcal{O})$ is composed of a Bose part 
$C_{+}$and a Fermi part $C_{-}$, given by

$$
C_{ \pm}=\frac{1}{2}\left(C \pm U_{0} C U_{0}\right)
$$

where $U_{0}=U_{0}^{*}=U_{0}^{-1}$ is the operator inducing the sign change of Fermi fields ("rotation by $2 \pi$ "). If $A \in \mathfrak{U}\left(\mathcal{O}_{a}\right)$ and $B \in \mathfrak{U}\left(\mathcal{O}_{b}\right)$ is any pair of operators associated with two arbitrary spacelike separated regions $\mathcal{O}_{a}$ and $\mathcal{O}_{b}$ there holds the commutator equation

$$
\left[A, B_{+}+U_{0} B_{-}\right]=0
$$

combining in compact form the normal spacelike commutation and anticommutation relations of Bose and Fermi operators, respectively.

The question of whether these locality properties persist in the transition from the Wightman fields $F_{\alpha}$ to the net $\mathcal{O} \rightarrow \mathfrak{Q}(\mathcal{O})$ is a subtle mathematical problem due to the fact that the field operators $F_{\alpha}(f)$ are unbounded. Several regularity conditions in terms of the fields are known which guarantee that no pathologies occur in this step (cf. [13] and references quoted therein). In the cases where the fields $F_{\alpha}$ generating the algebra $\mathfrak{P}$ are to be interpreted as observables, the locality property of the corresponding net of von Neumann algebras has to be demanded also on physical grounds. We therefore assume that the underlying Wightman theory is sufficiently regular and postulate

2. (Regularity) The net $\mathcal{O} \rightarrow \mathfrak{A}(\mathcal{O})$ defined in (3.1) satisfies the spacelike commutation relations (3.5).

It is a well-known consequence of the structure described so far that the vector $\Omega$ is cyclic and separating for the local algebras $\mathfrak{X}(\mathcal{O})$ : it is cyclic since the polynomials in the field operators $F_{\alpha}(f), f \in \mathscr{S}(\mathcal{O})$ generate a dense set of vectors from $\Omega$ according to the Reeh-Schlieder theorem [14]. From the locality condition (3.5), it then follows that $\Omega$ is also cyclic for $\mathfrak{X}(\mathcal{O})^{\prime}$, and hence separating for $\mathfrak{\mathfrak { l }}(\mathcal{O})$.

Another feature which is of interest here is the fact that the local net $\mathcal{O} \rightarrow \mathfrak{A}(\mathcal{O})$ can always be extended to a net $\mathcal{O} \rightarrow \overline{\mathfrak{A}}(\mathcal{O})$ which satisfies the condition of duality [15], and hence is the maximal net with the properties mentioned above. This net is obtained by first constructing for all open wedge-shaped regions $\mathscr{W}$ bounded by two characteristic planes algebras $\mathfrak{I}(\mathscr{W})$ as in relation (3.1). Then one defines the algebras $\overline{\mathfrak{A}}(\mathcal{O})$ associated with "causally convex" regions $\mathcal{O}$ (i.e. open, bounded regions, such as double cones, whose closure $\overline{\mathcal{O}}$ is the intersection of wedges $\mathscr{W}$ ), setting

$$
\overline{\mathfrak{A}}(\mathcal{O})=\bigwedge_{\mathscr{W} \supset \overline{\mathcal{O}}} \underline{\mathfrak{X}}(\mathscr{W}) .
$$

(An extension of this definition to arbitrary regions can be accomplished by additivity.) It is obvious from relation (3.6) that the algebras so defined are "continuous from the outside," i.e. if $\mathcal{O}_{i}$ is any decreasing family of regions such that $\bigcap_{i} \mathcal{O}_{i}=\overline{\mathcal{O}}$, we have

$$
\overline{\mathfrak{A}}(\mathcal{O})=\bigwedge_{i} \overline{\mathfrak{A}}\left(\mathcal{O}_{i}\right)
$$

The subsequent discussion applies to the "minimal net" defined in (3.1) as well as to the "maximal net" defined in (3.6). We therefore introduce the notation $\mathcal{O} \rightarrow \mathfrak{A}(\mathcal{O})$ for either one of these nets. 
We supplement now this general setting by two more specific assumptions distinguishing a physically significant class of models. Firstly, we assume the nuclearity condition employed in the previous section. We recall here in brief this postulate.

3. (Nuclearity) Let $\mathcal{O}$ be any fixed bounded region. Then the maps

$$
\Theta_{\beta}(A)=e^{-\beta H} A \Omega, \quad A \in \mathfrak{A}(\mathcal{O})
$$

are nuclear for any $\beta>0$. Moreover the trace-norms of these maps satisfy the bound in the limit $\beta \searrow 0$,

$$
\left\|\Theta_{\beta}\right\|_{1} \leqq e^{\left(\beta_{0} / \beta\right)^{n}},
$$

where $\beta_{0}, n$ are certain constants.

As already mentioned, this condition restricts the admissible number of local degrees of freedom of a theory. As a measure of this quantity one takes the energylevel density of the states which are well-localized inside the region $\mathcal{O}$. The quantity $\left\|\Theta_{\beta}\right\|_{1}$ is the analogue of the partition function in statistical mechanics, and the bound on $\left\|\Theta_{\beta}\right\|_{1}$ says that the level density should not grow substantially faster than that of an arbitrary number of indistinguishable particles confined to a box of finite volume. We note that in asymptotically free theories, where StephanBoltzmann's law can be applied at high temperatures, one expects that the constant $n$ appearing in the bound can be put equal to the dimension of space. For a more detailed discussion and justification of the nuclearity condition we refer to [3], cf. also [16].

The following result concerning the intrinsic structure of the local algebras is a straightforward consequence of the preceding discussion.

Proposition 3.1. The algebras $\mathfrak{A}(\mathcal{O})$ associated with causally convex regions $\mathcal{O}$ are hyperfinite.

Remark. In the case of the minimal net this statement holds in fact true for arbitrary regions, including unbounded ones such as $\mathscr{W}$.

Proof. It follows from the preceding assumptions and the analysis in Sect. 2 that the local net has the split property. Moreover, the algebras $\mathfrak{A}(\mathcal{O})$ are continuous from the inside (in the case of the minimal net) or from the outside (in the case of the maximal net), respectively. In the first case we choose a sequence of regions $\mathcal{O}_{i}$ such that $\mathcal{O}_{i} \subset \subset \mathcal{O}_{i+1}$ and $\bigcup_{i} \mathcal{O}_{i}=\mathcal{O}$. Then there exists an increasing family of type $I$ factors $\mathfrak{M}_{i}$ such that $\mathfrak{A}\left(\mathcal{O}_{i}\right) \subset \mathfrak{M}_{i} \subset \mathfrak{A}\left(\mathcal{O}_{i+1}\right)$, and hence

$$
\mathfrak{U}(\mathcal{O})=\bigvee_{i} \mathfrak{A}\left(\mathcal{O}_{i}\right)=\bigvee_{i} \mathfrak{M}_{i}
$$

Similarly, we find in the case of the maximal net a decreasing family of type $I$ factors $\mathfrak{N}_{i}$ such that

$$
\mathfrak{U}(\mathcal{O})=\bigwedge_{i} \mathfrak{N}_{i} .
$$

Since type $I$ factors on a separable Hilbert space $\mathscr{H}^{2}$ are clearly hyperfinite it is obvious from these approximation formulas that $\mathfrak{U}(\mathcal{O})$ is hyperfinite, too. (For an

${ }^{2}$ That $\mathscr{H}$ is separable is a trivial aspect of the nuclearity condition 
account of the standard properties of hyperfinite von Neumann algebras cf. [5].)

It remains to discuss the type of the local algebras. This property has been determined in [1] for local nets constructed from Wightman fields admitting a scaling limit. For the convenience of the reader we recall here the precise assumptions: given any positive, monotone function $N(\lambda), \lambda>0$ one first defines a scaling transformation of the testfunctions $f(x)$ on Minkowski space, setting

$$
f_{\lambda}(x)=N(\lambda) f(x / \lambda) \text {. }
$$

One then obtains a corresponding transformation of the field operators $F_{\alpha}(f)$ by replacing the testfunctions $f$ by $f_{\lambda}$. Now according to the theory of the renormalization group (cf. [17]) one expects that in renormalizable theories with an ultraviolet fixed point the vacuum expectation values of products of the scaled fields $F_{\alpha}\left(f_{\lambda}\right)$ have a non-trivial limit as $\lambda \searrow 0$ for an appropriate choice of the function $N(\lambda)$. In asymptotically free theories $N(\lambda)$ should be of the form $\lambda^{\delta-d}$ being the canonical dimension of the field and $d$ the dimension of Minkowski $[\delta$ space], possibly modified by logarithmic corrections. But the precise form of $N(\lambda)$ is irrelevant here. In fact one needs for the determination of the type of the local algebras only, the following mild assumption concerning the scaling properties of the underlying Wightman theory.

4. (Asymptotic scale invariance) Amongst the Wightman fields $F_{\alpha}$ generating the local net $\mathcal{O} \rightarrow \mathfrak{U}(\mathcal{O})$ there is some field $F$ with vanishing vacuum expectation value such that for a suitable choice of $N(\lambda)$ the scaled field operators $F\left(f_{\lambda}\right)$ have the following properties: the expectation values $\left(\Omega, F\left(f_{\lambda}\right)^{*} F\left(f_{\lambda}\right) \Omega\right)$ converge for all testfunctions $f$ in the limit $\lambda \searrow 0$ and are nonzero for some $f$, and the norms $\left\|F\left(f_{\lambda}\right)^{*} F\left(f_{\lambda}\right) \Omega\right\|$ and $\left\|F\left(f_{\lambda}\right) F\left(f_{\lambda}\right)^{*} \Omega\right\|$ stay bounded in this limit.

Combining this input with information about the modular groups associated with the algebras $\mathfrak{U}(\mathscr{W})$ and the vacuum state $\Omega$ [15], one can determine the Connes invariant of the local algebras, and hence their type. This was demonstrated in [1] under the assumption that the field $F$ having the required scaling properties transforms as a scalar under Lorentz-transformations. But it is evident from that argument that the conclusions hold just as well if $F$ is finitely covariant. So there holds the

Proposition 3.2. The algebras $\mathfrak{A}(\mathcal{O})$ associated with causally convex regions are of type $I I I_{1}$, i.e. only factors of type $I I I_{1}$ appear in the central decomposition of $\mathfrak{A}(\mathcal{O})$.

Remark. Again, one can extend this result to algebras associated with a larger class of regions, cf. [1].

In case that the local algebras are factors we thus have reached our goal: according to the result of Haagerup already quoted, the hyperfinite type $I I I_{1}$ factor $\mathfrak{R}$ is unique, so all local algebras are isomorphic to $\mathfrak{R}$. Taking also into account the possibility that the local algebras have a center we can summarize the results of this discussion in the following

Theorem 3.3. Let $\mathcal{O} \rightarrow \mathfrak{U}(\mathcal{O})$ be a (minimal or maximal) local net constructed from a Wightman theory which satisfies the conditions of nuclearity and asymptotic scale 
invariance. Then the algebras $\mathfrak{A}(\mathcal{O})$ associated with causally convex regions $\mathcal{O}$ are isomorphic to $\mathfrak{R} \otimes 3$, where $\mathfrak{R}$ is the unique hyperfinite factor of type $I I I_{1}$ and 3 the center of $\mathfrak{U}(\mathcal{O})$.

Proof. Since in the central decomposition of a hyperfinite von Neumann algebra there appear only hyperfinite factors [5], it follows from the preceding two propositions that $\mathfrak{U}(\mathcal{O})$ can be expressed as a direct integral $\mathfrak{U}(\mathcal{O})=\int d \mu(z) \mathfrak{R}(z)$ of hyperfinite type $I I I_{1}$ factors $\mathfrak{R}(z)$. Because of the uniqueness of this factor it is then clear (cf. [11, Chap. 4.5]) that $\mathfrak{A}(\mathcal{O})$ is isomorphic to $\mathfrak{R} \bar{\otimes} \mathfrak{3}$.

\section{References}

1. Fredenhagen, K.: On the modular structure of local algebras of observables. Commun. Math. Phys. 97, 79 (1985)

2. Haagerup, U.: Connes' bicentralizer problem and uniqueness of the injective factor of type $I I I_{1}$ (to appear in Acta Math.)

3. Buchholz, D., Wichmann, E.H.: Causal independence and the energy level density of states in local quantum field theory. Commun. Math. Phys. 106, 321 (1986)

4. Haag, R., Schroer, B.: Postulates of quantum field theory. J. Math. Phys. 3, 248 (1962)

5. Connes, A.: Classification des facteurs. In: Proceedings of Symposia in Pure Mathematics Vol. 38/2. Providence, RI: Am. Math. Society 1982

6. Longo, R.: Algebraic and modular structure of von Neumann algebras of physics. In: Proceedings of Symposia in Pure Mathematics Vol. 38/2. Providence, RI: Am. Math. Society 1982

7. Doplicher, S., Longo, R.: Standard and split inclusions of von Neumann algebras. Invent. Math. 73, 493 (1984)

8. Doplicher, S.: Local aspects of superselection rules. Commun. Math. Phys. 85, 73 (1982)

9. Takesaki, M.: Theory of operator algebras. I. Berlin, Heidelberg, New York: Springer 1979

10. Jaffe, A.M.: High-energy behavior in quantum field theory. Phys. Rev. 158, 1454 (1967)

11. Sakai, S.: $C^{*}$-algebras and $W^{*}$-algebras. Berlin, Heidelberg, New York: Springer 1971

12. Streater, R.F., Wightman, A.: PCT, spin and statistics, and all that. New York: Benjamin 1964

13. Driessler, W., Summers, St.J., Wichmann, E.H.: On the connection between quantum fields and von Neumann algebras of local operators. Commun. Math. Phys. 105, 49 (1986)

14. Reeh, H., Schlieder, S.: Bemerkungen zur Unitäräquivalenz von Lorentz-invarianten Feldern. Nuovo Cimento 22, 1051 (1961)

15. Bisognano, J.J., Wichmann, E.H.: On the duality condition for quantum fields. J. Math. Phys. 17, 303 (1976)

16. Buchholz, D., Jacobi, P.: On the nuclearity condition for massless fields (to appear in Lett. Math. Phys.)

17. Wilson, K.G.: Renormalization group and strong interactions. Phys. Rev. D 3, 1818 (1971)

Communicated by K. Osterwalder

Received December 18, 1986 
